\title{
Sala de vacina: organização dos imunobiológicos e práticas profissionais
}

\author{
Vaccine room: organization of immunobiologicals and \\ professional practices
}

\author{
Camilla Xavier Cunha' $\bullet$ Thaina Jacome Andrade de Lima ${ }^{2} \bullet$ Maria Valéria Chaves de Lima ${ }^{3}$ \\ Kalyane Kelly Duarte de Oliveira ${ }^{4}$ Ilza Iris dos Santos ${ }^{5}$
}

\begin{abstract}
RESUMO
OBJETIVO: analisar a organização e as práticas profissionais nas salas de vacinas de um município de médio porte. METODOLOGIA: trata-se de uma pesquisa descritiva, de abordagem quantitativa. A população foi composta por 09 técnicos de enfermagem de 09 unidades básicas de saúde. Os instrumentos utilizados para coleta de dados foram check list adaptado a partir do formulário disponibilizado pelo Programa de Avaliação do Instrumento de Supervisão Sala de Vacinação - PAISSV. Os dados foram tabulados em planilha eletrônica e transferidos para o programa estatístico SPSS (Statistical Package for the Social Sciences) versão 23.0, os quais foram expressos em frequência simples e porcentagem, bem como média \pm desvio padrão, mínimos e máximos. RESULTADOS: Os profissionais participantes da pesquisa foram técnicos de enfermagem, do sexo feminino. Os dados descritivos, estão relacionados aos aspectos gerais da sala de vacina; condições de conservação; estoque dos insumos; as condições dos termômetros utilizados; e as forma de armazenamento dos insumos. CONSIDERAÇÕES FINAIS: evidenciou-se que as recomendações previstas no Manual de Rede de Frio não estão em sua total conformidade com a prática de conservação de vacinas nas UBSs do município, bem como o serviço local apresenta deficiências que podem interferir na efetividade do Programa Nacional de Imunização.

Palavras-chaves:Vacinas; Programa Nacional de Imunização; Enfermagem.
\end{abstract}

\begin{abstract}
OBJECTIVE: to analyze the organization and professional practices in the vaccination rooms of a medium-sized municipality. METHODOLOGY: this is a descriptive research, with a quantitative approach. The population consisted of 09 nursing technicians from 09 basic health units. The instruments used for data collection were a checklist adapted from the form provided by theVaccination Room Supervision Instrument Evaluation Program - PAISSV.The data were tabulated in an electronic spreadsheet and transferred to the statistical program SPSS (Statistical Package for the Social Sciences) version 23.0, which were expressed as simple frequency and percentage, as well as mean \pm standard deviation, minimum and maximum. RESULTS:The professionals participating in the research were female nursing technicians. The descriptive data are related to the general aspects of the vaccine room; conservation conditions; input stock; the conditions of the thermometers used; and the ways of storing the inputs. FINAL CONSIDERATIONS: it became evident that the recommendations provided for in the Cold Chain Manual are not in full compliance with the vaccine conservation practice in the UBSs of the municipality, as well as the local service has deficiencies that may interfere with the effectiveness of the National Health Program. Immunization.
\end{abstract}

Keywords:Vaccines; National Immunization Program; Nursing.

I Bacharel em Enfermagem pela Faculdade Nova Esperança de Mossoró - FACENE/RN camillaxaviercunha@hotmail.com - https://orcid.org/0000-0003-1017-2343

2 Acadêmica de Enfermagem da Universidade do Estado do Rio Grande do Norte-UERN, Campus Avançado de Pau dos Ferros- CAPF - thainajacome@hotmail. com - https://orcid.org/0000-0003-1289-8842

3 Acadêmica de Enfermagem da Universidade do Estado do Rio Grande do Norte-UERN, Campus Avançado de Pau dos Ferros- CAPF valerialimal3@hotmail. com - https://orcid.org/0000-0002-9278-5612

4 Doutora pelo Programa de Pós- Graduação em Enfermagem da Universidade Federal do Rio Grande do Norte- UFRN, Professora da Universidade do Estado do Rio Grande do Norte-UERN, Campus Avançado de Pau dos Ferros- CAPF kenfoliveira@gmail.com - https://orcid.org/0000-000 I-77/3-3264

5 Prof visitante na Faculdade de Ensino Integrado ASLIM. Enfermagem pela Universidade Potiguar-UnP - ilzairis@hotmail.com - https://orcid.org/0000-0003-3886-7470 


\section{INTRODUÇÃO}

A imunização compõe uma das estratégias mais eficazes de prevenção de doenças e requer conhecimento adequado que garanta sua qualidade e efetividade, por isso, há uma grande preocupação para que essa imunização aconteça de forma efetiva. Diante disso, a Atenção Primária à Saúde (APS) tornou essa uma de suas ações prioritárias, tendo em vista sua perspectiva de entrada principal aos serviços de saúde, bem como, seu perfil de promoção, recuperação e prevenção a saúde. Embora imunizar seja uma ação que perpassa pelo nível municipal, estadual e federal, pelo fato da atenção básica estar mais propícia aos municípios, por meio da descentralização, as unidades básicas de saúde acabam se tornando o local onde essa imunização se concretiza. ${ }^{(1)}$

A estrutura da Rede de Frio permeia as três esferas administrativas organizando-se em instâncias Nacional, Estadual, Regional, Municipal e Local com fluxos de distribuição e armazenamento basicamente verticalizados (os imunobiológicos são distribuídos na seguinte ordem: Nacional distribui para o Estado, que entrega para as Regionais, que repassam para os Municípios, que por fim, chegam as UBS Locais). Contudo, a depender de situações epidemiológicas e/ou emergenciais específicas, podem ocorrer de forma horizontalizada. (2)

Os imunobiológicos disponibilizados no serviço de vacinação devem ser mantidos em condições adequadas de transporte, armazenamento e distribuição, permitindo que eles permaneçam com suas características iniciais até o momento da sua administração. Alterações de temperatura, excesso de frio ou calor, podem comprometer a potência imunogênica, o que pode acarretar a redução ou a falta do efeito esperado. Os imunobiológicos, enquanto produtos termolábeis e/ou fotossensíveis, necessitam de armazenamento adequado para que suas características imunogênicas sejam mantidas, devendo ser conservadas entre $+2^{\circ} \mathrm{C} \mathrm{e}+8^{\circ} \mathrm{C}$ para não perder sua eficácia. Tamanha é a importância destes fatores sobre a manutenção da qualidade dos imunobiológicos, que sempre foi objeto de norma técnica do $\mathrm{PNI}$, constituindo um manual específico: Manual de Rede de Frio. (2)

Nas Unidades Básicas de Saúde (UBS), segundo a decisão $n^{\circ} 42$, de 24 de maio de 2018 , as atividades da sala de vacinação devem ser desenvolvidas pela equipe de enfermagem treinada e capacitada para os procedimentos de manuseio, conservação, preparo e administração, registro e descarte dos resíduos resultantes das ações de vacinação. Sendo o enfermeiro é responsável pela supervisão ou pelo monitoramento do trabalho desenvolvido na sala de vacinação, como também pelo processo de educação permanente da equipe. ${ }^{(3)}$

Nesse contexto suscita-se os seguintes questionamentos: Como é realizada a organização dos imunobio- lógicos nas Salas de Vacinas do município de Mossoró/ RN? Quais as práticas profissionais em relação a conservação dos imunobiológicos nas UBS?

O estudo tem como hipótese que o município de Mossoró/RN mantém as recomendações necessárias para armazenamento dos imunobiológicos nas UBS, a fim de evitar possíveis perdas ou até a não imunização por falhas no armazenamento e organização deles. Não podendo esquecer, a importância dos profissionais, pois são eles que mantêm os imunobiológicos dentro dos padrões estabelecidos, e o conhecimento relacionado a tais práticas.

Diante do exposto, visto que o profissional de enfermagem é o responsável pelas ações de imunização, desde a conservação até a administração das vacinas, o presente estudo justifica-se pela necessidade de avaliar a organização e as práticas de conservação dos imunobiológicos nas Salas de Vacina, tendo em vista também o conhecimento dos profissionais de enfermagem, para compreender este processo.

A escolha dessa temática de pesquisa, justifica-se pelas vivências experimentadas em uma rede de frio e sala de vacina, nos quais, éramos cobrados constantemente quanto à organização e condições de armazenamento dos imunobiológicos, seja no refrigerador ou nas caixas térmicas.

Dessa forma, espera-se que o estudo venha contribuir para o conhecimento relacionado à Sala de Vacina (organização de imunobiológicos e a conservação), diante da importância da educação permanente em saúde para os profissionais de enfermagem. Assim, o objetivo deste trabalho é analisar a organização e as práticas profissionais nas salas de vacinas de um município de médio porte.

\section{METODOLOGIA}

Trata-se de uma pesquisa descritiva, de abordagem quantitativa. A pesquisa foi desenvolvida na cidade de Mossoró, situada no estado do Rio Grande do Norte. Atualmente a cidade dispõe de 49 unidades básicas de saúde, sendo 42 localizadas na zona urbana e 7 na zona rural, caracterizando-se como a população da pesquisa.A amostragem da pesquisa foi definida de forma não probabilística, de forma que as unidades básicas de saúde foram selecionadas por serem campos de práticas da faculdade em que estava vinculada a pesquisadora responsável e participante. As unidades que fizeram parte da amostra localizam-se em diversas regiões do município, sendo 8 de zona urbana e I de zona rural. Assim, a amostra do estudo foi composta por um total de 9 unidades básicas de saúde e 9 técnicos de enfermagem que trabalhavam nas salas de vacina das unidades.

Participaram da pesquisa somente as unidades que ti- 
nham a sala de vacinas funcionando e com profissionais atuantes na unidade com no mínimo de 6 meses de exercício da profissão, sendo excluídas da amostra as unidades de saúde com sala de vacina fechada, os profissionais que estavam de férias, licença e, com menos de 6 meses de exercício da profissão.

O instrumento utilizado para coleta de dados foi um check list adaptado a partir do formulário disponibilizado pelo Programa de Avaliação do Instrumento de Supervisão Sala de Vacinação - PAISSV, neste, foi realizado adaptações, pois ele não contemplava mais o calendário vacinal em vigor. ${ }^{(4)}$

O checklist foi preenchido pelo pesquisador através de observação dos equipamentos da rede de frio e respostas dadas oralmente pelos responsáveis pelas salas de vacinas. Envolvendo questões de como está a organização dos equipamentos da rede de frio, materiais necessários para a sala de vacina. A coleta foi realizada de fevereiro a maio de 2018.

Para a realização da coleta de dados, efetuou se a articulação com a coordenação de imunobiológicos do município, Atenção Básica e enfermeiros, responsáveis pela Estratégia Saúde da Família do município, abordando o principal objetivo da pesquisa. A coleta iniciou-se após a aprovação pelo comitê de ética, sendo realizada no primeiro momento, uma visita às unidades, sendo entregue e explicado a cada profissional a pesquisa e o termo de Consentimento Livre e Esclarecido (TCLE), para dessa forma garantir que a participação voluntária e em concordância dos termos do TCLE.

Os dados foram tabulados em planilha eletrônica e transferidos para o programa estatístico SPSS (Statistical Package for the Social Sciences) versão 23.0, os quais foram expressos em frequência simples e porcentagem, bem como média \pm desvio padrão, mínimos e máximos. Sempre quando necessário as variáveis sofreram transformação do tipo dummy.

A pesquisa foi submetida e aprovada no Comitê de Ética da Faculdades Nova Esperança - FACENE, sob parecer $n^{\circ} 61 / 2017$ (CNS).

\section{RESULTADOS}

Os principais resultados dados encontrados são as características dos profissionais, sendo elas, sexo, idade, tempo de atuação em sala de vacina, e localização da unidade de saúde (área urbana ou rural).

Tabela 1 - Caracterização dos profíssionais e sala de vacina. Mossoró-RN, Brasil. 2017

Variáveis $\quad$ Freq. $\%$

\section{Idade (anos)}

Até 40

Média \pm desvio padrão

Mínimo - Máximo

Tempo de atuação na sala de vacina (anos)

Até 02

03 a 10

Média \pm desvio padrão

Mínimo - Máximo

Área

Urbana

Rural
$41,0 \pm 11,0$

$25-58$
$\%$

(20) 
Tabela 2 - Aspectos gerais da sala de vacinação. Mossoró-RN, Brasil. 2017

\begin{tabular}{|c|c|c|}
\hline Variáveis & Freq. & $\%$ \\
\hline \multicolumn{3}{|l|}{ Exclusiva para esta atividade } \\
\hline Sim & 03 & 33,3 \\
\hline Não & 06 & 66,7 \\
\hline \multicolumn{3}{|l|}{ Condições ideais de conservação } \\
\hline $\operatorname{Sim}$ & 02 & 22,2 \\
\hline Não & 07 & 77,8 \\
\hline \multicolumn{3}{|c|}{ Temperatura ambiente entre $+18^{\circ} \mathrm{C} \mathrm{e}+20^{\circ} \mathrm{C}$} \\
\hline $\operatorname{Sim}$ & 09 & 100,0 \\
\hline Não & 0 & 0,0 \\
\hline \multicolumn{3}{|l|}{ Observa a validade da vacina } \\
\hline Sim & 09 & 100,0 \\
\hline Não & 0 & 0,0 \\
\hline \multicolumn{3}{|l|}{ Quantitativo suficiente para a demanda } \\
\hline $\operatorname{Sim}$ & 07 & 77,8 \\
\hline Não & 02 & 22,2 \\
\hline \multicolumn{3}{|l|}{ Estoque excessivo de vacinas } \\
\hline $\operatorname{Sim}$ & 02 & 22,2 \\
\hline Não & 07 & 77,8 \\
\hline \multicolumn{3}{|c|}{$\begin{array}{l}\text { Indicação na caixa de distribuição elétrica para não desligar o } \\
\text { disjuntor da sala de vacina }\end{array}$} \\
\hline Sim & 01 & 11,1 \\
\hline Não & 08 & 88,9 \\
\hline \multicolumn{3}{|l|}{ Funcionamento da sala de vacina } \\
\hline Manhã & 01 & 11,1 \\
\hline Tarde & 0 & 0,0 \\
\hline Manhã e Tarde & 08 & 88,9 \\
\hline
\end{tabular}

Fonte: Dados da pesquisa (2017).

Outros dados descritivos, estão relacionados aos aspectos gerais da sala de vacina das unidades de saúde, sendo trazido condições de conservação e estoque dos insumos.

Outros dados apresentados são referentes a rede de frio. A tabela 3 traz as condições do refrigerador.

Já a tabela 4, traz outros dados referentes a rede de frio, as condições dos termômetros encontrados nas unidades de saúde.

Por último, a tabela 5 ainda sobre a rede de frio, traz as formas de armazenamento dos insumos nas unidades de saúde participantes da pesquisa.

\section{DISCUSSÃO}

A tabela I demonstra a característica dos profissionais da sala de vacina, que predomina o sexo feminino, o tempo de atuação na sala de vacina variou de até 2 anos a acima de 10 anos, com média igual a $11,0 \pm 10,0$ e com idade máxima de atuação de 30anos.

Vale salientar que a eficiência do trabalho realizado assim como o cumprimento das metas previstas vai muito além do número de profissionais que exercem funções na sala de vacina. Leva-se em consideração primordialmente a quantidade de capacitações que estes 
Tabela 3 - Aspectos gerais da Rede de frio: Refrigerador. Mossoró-RN, Brasil. 2017

\begin{tabular}{lcr}
\hline \multicolumn{1}{c}{ Variáveis } & Freq. & $\%$ \\
\hline Tomada elétrica exclusiva para o refrigerador & & 88,9 \\
Sim & 08 & 11,1, \\
Não & 01 & \\
O refrigerador é exclusivo para os imunobiológicos & & 66,7 \\
Sim & 06 & 33,3 \\
Não & 03 &
\end{tabular}

Temperatura do refrigerador entre $+2^{\circ} \mathrm{C}$ e $+8^{\circ} \mathrm{C}$

Sim

09

100,0

Não

Capacidade do refrigerador igual ou superior a $280 \mathrm{~L}$

$\operatorname{Sim}$

Não

Refrigerador em bom estado de conservação

Sim

Não

Refrigerador em estado ideal de funcionamento

Sim

Não

100,0

09

Refrigerador em estado ideal de limpeza

Sim

Não

Refrigerador está distante da fonte de calor

Sim

Não

09

100,0

Refrigerador distante de luz solar direta

Sim

Não

Refrigerador com $20 \mathrm{~cm}$ da parede

Sim

55,6

Não

Quantidade de bobinas de gelo recomendada no evaporador

Sim

Bandeja coletora de água no refrigerador

Garrafas de água com corante no espaço inferior do refrigerador Sim 55,6 Não

Mantém o material na porta do refrigerador

Sim

Não

$O$ refrigerador do tipo frost-free

Sim

Não

Fonte: Dados da pesquisa (2017). 
Tabela 4 - Aspectos gerais da Rede de frio: Termômetro. Mossoró-RN, Brasil. 2017

\begin{tabular}{|c|c|c|}
\hline Variáveis & Freq. & $\%$ \\
\hline \multicolumn{3}{|l|}{ Termômetro digital no refrigerador } \\
\hline Sim & 09 & 100,0 \\
\hline Não & 0 & 0,0 \\
\hline \multicolumn{3}{|c|}{$\begin{array}{l}\text { Faz a leitura e o registro da temperatura no início e fim da } \\
\text { jornada }\end{array}$} \\
\hline Sim & 08 & 88,9 \\
\hline Não & 01 & 11,1 \\
\hline \multicolumn{3}{|l|}{ Mapa de controle diário em local visível } \\
\hline $\operatorname{Sim}$ & 06 & 66,7 \\
\hline Não & 03 & 33,3 \\
\hline \multicolumn{3}{|c|}{ Temperatura da caixa térmica entre $+2^{\circ} \mathrm{C}$ e $+8^{\circ} \mathrm{C}$} \\
\hline $\operatorname{Sim}$ & 06 & 66,7 \\
\hline Não & 03 & 33,3 \\
\hline \multicolumn{3}{|c|}{ Monitora a temperatura da caixa térmica de uso diário } \\
\hline $\operatorname{Sim}$ & 05 & 55,6 \\
\hline Não & 04 & 44,4 \\
\hline \multicolumn{3}{|c|}{ Termômetro suficiente para atividades de rotina } \\
\hline Sim & 08 & 88,9 \\
\hline Não & 01 & 11,1 \\
\hline
\end{tabular}

Fonte: Dados da pesquisa (2017).

participam com o intuito de melhorar suas técnicas e suas atitudes. A prova viva disso, são as orientações do próprio ministério da saúde que incentiva uma capacitação continuada e no próprio ambiente de trabalho, visando metodologias ativas e interdisciplinares e que surgem das experiências dos trabalhadores em seu dia a dia. ${ }^{(5)}$

A sala de vacina tem diversas atividades para serem desenvolvidas, porém, o presente estudo detém-se ao trabalho do técnico de enfermagem, que na prática atuam como vacinadores, sendo supervisionados pelo enfermeiro que responde também pela parte técnica da sala.

O Manual de Normas e Procedimentos para Vacinação/PNI traz em seus Aspectos Técnicos e Administrativos da Atividade de Vacinação que a vacinação para acontecer deve possuir uma equipe própria e com funções básicas. Essa equipe deve ser constituída no mínimo por um Enfermeiro e técnico ou auxiliar de enfermagem, bem como possuir dois vacinadores a cada turno de trabalho. A quantidade da equipe vai ser estipulada pela demanda populacional e de serviço do território a qual a unidade cobre. Outra forma de também se ter noção do dimensionamento da equipe é realizando se a pro- porção de que um vacinador pode injetar 90 doses de vacina oral ou 30 doses de vacina injetável por hora de trabalho. ${ }^{(6)}$

Segundo a lei de $n^{\circ} 7.498 / 86$, regulamentada pelo decreto 94.406/87, em seu Art. 15, têm se: "Atividades referidas nos artigos 12 e 13 desta lei, quando exercidas em instituições de saúde, pública e privadas, e em programas de saúde, somente podem ser desempenhadas sob orientação e supervisão de enfermeiro". ${ }^{(7)}$

$\mathrm{Na}$ supervisão é exigido ao enfermeiro a Responsabilidade Técnica (RT) pelo serviço, o que está estabelecido na Resolução n 302 de 2005 do Conselho Federal de Enfermagem. ${ }^{(8)}$ Sendo assim, tal dispositivo traz a afirmativa de que todas as atividades de enfermagem desempenhadas pelo técnico de enfermagem e auxiliar de enfermagem, somente poderão ser desenvolvidas sob orientação e supervisão do enfermeiro.

Um fator relevante que a tabela 2 demonstra, é que $66,7 \%$ dos profissionais usam a sala de vacina para desenvolver outras atividades que não estejam relacionadas aos procedimentos de vacinação de rotina, campanhas, bloqueios e intensificações. Conforme o Manual de Nor- 
Tabela 5 - Aspectos gerais da Rede de frio: Armazenamento. Mossoró-RN, Brasil. 2017

\begin{tabular}{|c|c|c|}
\hline Variáveis & Freq. & $\%$ \\
\hline \multicolumn{3}{|c|}{$\begin{array}{l}\text { Na } 1^{\text {a }} \text { prateleira do refrigerador: vacinas que podem ser } \\
\text { submetidas à temperatura negativa }\end{array}$} \\
\hline $\operatorname{Sim}$ & 06 & 66,7 \\
\hline Não & 03 & 33,3 \\
\hline \multicolumn{3}{|c|}{$\begin{array}{l}\text { Na } 2^{\mathrm{a}} \text { prateleira do refrigerador: vacinas que não ser } \\
\text { submetidas à temperatura negativa }\end{array}$} \\
\hline $\operatorname{Sim}$ & 06 & 66,7 \\
\hline Não & 03 & 33,3 \\
\hline \multicolumn{3}{|c|}{$\begin{array}{l}\text { Na } 3^{\text {a }} \text { prateleira do refrigerador: estoque de vacinas, soros e } \\
\text { diluentes }\end{array}$} \\
\hline $\operatorname{Sim}$ & 06 & 66,7 \\
\hline Não & 03 & 33,3 \\
\hline \multicolumn{3}{|c|}{ Vacinas organizadas por tipo, lote e validade } \\
\hline Sim & 03 & 33,3 \\
\hline Não & 06 & 66,7 \\
\hline \multicolumn{3}{|c|}{ Mantém distância entre as vacinas e a parede do refrigerador } \\
\hline $\operatorname{Sim}$ & 0 & 0,0 \\
\hline Não & 09 & 100,0 \\
\hline \multicolumn{3}{|c|}{ Caixa térmica suficiente para demanda } \\
\hline $\operatorname{Sim}$ & 07 & 77,8 \\
\hline Não & 02 & 22,2 \\
\hline \multicolumn{3}{|c|}{ Bobinas de gelo suficiente para demanda } \\
\hline $\operatorname{Sim}$ & 08 & 88,9 \\
\hline Não & 01 & 11,1 \\
\hline \multicolumn{3}{|c|}{ Faz a ambientação das bobinas de gelo } \\
\hline $\operatorname{Sim}$ & 01 & 11,1 \\
\hline Não & 08 & 88,9 \\
\hline
\end{tabular}

Fonte: Dados da pesquisa (2017).

mas e Procedimentos de Vacinação, a sala de vacinação é classificada como área semicrítica e deve ser destinada exclusivamente à administração dos imunobiológicos, é importante que todos os procedimentos desenvolvidos promovam a máxima segurança, reduzindo o risco de contaminação para os indivíduos vacinados e para a equipe de vacinação. ${ }^{(6)}$

É indispensável também nesta tabela, observar que $77,8 \%$ das salas de vacinas não se enquadram ao que é preconizado pelo Manual de Normas e Procedimentos para Vacinação (20l4), que orienta como deve ser a estrutura física da sala de vacina a fim de manter e favorecer a conservação dos imunobiológicos e evitar possíveis contaminações.As salas de vacina evidenciaram situações multifárias como, tamanho da sala de vacina diminuto para a demanda, paredes descascadas, piso comum, teto com infiltração, somente uma pia com balcão para realizar todos os procedimentos (lavagem das mãos, dos materiais e preparo das vacinas). ${ }^{(6)}$

É impreterível a tomada de providências para que estas situações citadas acima sejam regulamentadas segundo o Manual de Normas e Procedimentos para Vacinação (20I4), o qual determina que a área mínima seja de $6 \mathrm{~m}^{2}$, contudo, recomenda-se uma área média a partir de 9 $\mathrm{m}^{2}$ para a adequada disposição dos equipamentos e dos mobiliários e o fluxo de movimentação em condições ideais para a realização das atividades. Piso e paredes lisos, contínuos (sem frestas) e laváveis. Teto com acaba- 
mento resistente à lavagem. Duas pias (uma para lavagem dos materiais e outra para lavagem das mãos) e bancada feita de material não poroso para preparo dos insumos, dentre outros requisitos a fim de assegurar a qualidade da assistência prestada. ${ }^{(6)}$

Concluindo esta análise, é conveniente apontar que $88,9 \%$ das unidades não apresentam indicação na caixa de distribuição elétrica para não desligar o disjuntor da sala de vacina, esta indicação tem o intuito de garantir a conservação das vacinas dentro dos padrões estabelecidos e evitar possíveis perdas de imunobiológicos.

Pois, um dos principais problemas da efetividade das vacinas hoje está relacionada com a má conservação dos imunobiológicos diretamente relacionada com a rede de frios. Caracterizando-se por episódios de desligamento inadequado, mau posicionamento dos compostos, não exclusividade do disjuntor, limpeza e temperaturas inadequadas. ${ }^{(9)}$

A tabela 3 mostra que 33,3\% das unidades utilizam o refrigerador para armazenar outros produtos (insulina, PPD, produtos odontológicos, teste do pezinho), além dos imunobiológicos, violando a recomendação feita pelo $\mathrm{PNI}$ de que o refrigerador é de uso exclusivo para o armazenamento dos imunobiológicos, uma vez que, o armazenamento de outros materiais no refrigerador de vacinas expõe as mesmas a mais riscos, tanto de contaminação, devido ao aumento da manipulação do equipamento, quanto de maior exposição e oscilação à variação da temperatura. ${ }^{(10)}$

Mesmo apresentando um índice de II,I\% referente ao uso do refrigerador doméstico com capacidade inferior a 280 litros, é importante ressaltar que o Manual da Rede de Frio/PNI proibiu o uso destes em 2017.

Nas UBSs deste estudo, foram observadas as temperaturas do refrigerador no momento da visita e em todas elas estavam de acordo com os parâmetros de segurança para a conservação.

Quanto a parte interna do refrigerador, 44,4\% não possuem garrafas de água com corante dentro da gaveta (parte de baixo do refrigerador), estas são fundamentais por formarem uma massa térmica que promove a recuperação mais rápida da temperatura. Ainda na parte interna do refrigerador, $88,9 \%$ mantêm os materiais na porta da geladeira, estes precisam ser retirados para que haja uma melhor circulação de ar frio, e que a temperatura na qual atinge as vacinas seja a correta. Em relação ao tipo de refrigerador utilizado, comprovou-se que $44,4 \%$ são do tipo frost-free. A Organização Mundial de Saúde - veda o uso deste tipo de refrigerador para o armazenamento de imunobiológicos, pois não tem efetividade do rendimento e a espessura do isolamento das paredes facilita a troca de calor com o meio externo. Desta forma vê se que há uma falha considerável da organização da rede de frios que pode comprometer diretamente a qualidade desses imunizantes. ${ }^{(9)}$

A tabela 4 evidencia que 100,0\% das UBSs fazem o uso correto do termômetro no refrigerador, o qual é indispensável para este tipo de refrigerador já que seu uso não é mais indicado por não dispor de um valor preciso no ajuste da temperatura.

Em relação a temperatura correta das caixas térmicas, $33,3 \%$ apresentavam no momento da visita uma temperatura imprópria para as vacinas e que $44,4 \%$ não controlam a temperatura das caixas térmicas, o que explica o resultado anterior e afeta na qualidade e eficácia das vacinas por serem produtos termolábeis e/ou fotossensíveis.A importância da verificação da temperatura das caixas térmicas justifica-se pelo fato de as bobinas de gelo funcionarem como receptor de calor do ar e das vacinas. Dessa forma, quando todo o calor for transferido para o gelo, este começará a derreter-se, necessitando da troca de novas bobinas, o que não ocorrerá se esta mudança não for monitorada, não devendo administrar as vacinas sem a certificação da conservação adequada. (II)

Nesta tabela 5 nota-se que tanto na primeira prateleira do refrigerador quanto na segunda prateleira, 33,3\% não têm o cuidado em expor os imunobiológicos a temperaturas as quais eles são sensíveis, o mesmo acontece com a ambientação das bobinas de gelo, revelando que $88,9 \%$ dos profissionais não a realizam. Ao retirar as bobinas do congelador ou freezer elas chegam a aproximadamente $-7^{\circ} \mathrm{C}$. Atualmente os estudos apontam o congelamento de vacinas nas diversas fases da rede de frio, o que resulta na perda de potência comprometendo a imunogenicidade. (II)

Evidencia-se também que $66,7 \%$ não armazenam as vacinas por tipo, lote ou validade, a fim de evitar a perda desses imunobiológicos por vencimento deles. Durante observação da distância correta dos imunobiológicos e as paredes do refrigerador foram observados em 100,0\% de descumprimento do item que tem como objetivo, perdurar a corrente de ar similar entre os imunobiológicos, conforme recomendado pela Rede de Frio/PNI. ${ }^{(2)}$

A enfermagem exerce um papel fundamental nas ações de execução do Programa Nacional de Imunização, sendo de sua total responsabilidade prover periodicamente as necessidades de materiais para manter as condições ideais de conservação dos imunobiológicos e também, manter os equipamentos em boas condições de funcionamento tendo em vista a importância da vacinação para a vida de milhares de pessoas. E o enfermeiro como coordenador desta equipe deve estar preparado para orientar, incentivar, despertar interesse para garantir uma assistência segura, deve estar apto para procurar os faltosos e estar atualizado quanto as novas normas para transmitir aos demais a prática efetiva. ${ }^{(12)}$ 
É perceptível que o enfermeiro responsável por uma Unidade Básica de Saúde dispõe de várias atribuições que, por vezes, devido à grande demanda da unidade como serviços prestados à população, o impede de cumpri-las à risca como pede o Ministério da Saúde. Esta situação não é diferente com os técnicos de enfermagem, dificultando assim, de seguir o que é preconizado pelo Manual de Normas e Procedimentos para Vacinação, incumbência que o enfermeiro deve ter em realizar o dimensionamento dos profissionais para que não interfira nas ações prestadas à população. ${ }^{(6)}$

No entanto, não é somente a sobrecarga de tarefas que impossibilita estes profissionais de desempenharem com êxito suas funções, a gestão precisa olhar para a carência que vive as unidades no presente momento, seja pelos materiais essenciais de consumo utilizados na sala de vacina para conservação e armazenamento dos imunobiológicos, a escassez de profissionais, como a importância da educação permanente em saúde, que visa aprimorar o método educacional em saúde, tendo o processo de trabalho como seu objeto de transformação, com o intuito de melhorar a qualidade dos serviços, visando alcançar equidade no cuidado e tornando-os mais qualificados para $\circ$ atendimento das necessidades da população.

Em complemento a isso, as informações ou mitos em relação a vacinas são também empecilhos para que a vacinação alcance seu real proposito de proteção e prevenção. A cobertura vacinal tem sofrido quedas, devido a insegurança dos pais em relação a segurança do processo. Portanto perpassa-se ao profissional coordenador do setor de vacinação a função de explicar a importância e como acontece o processo de vacinação a população a qual ele é responsável. Deve se destacar que embora seja um processo meramente desconfortável é primordial, para a erradicação de doenças. Desta forma as ações devem ser pautadas no diálogo e incentivo, para que com a população ativa e informada, e disposta a se vacinar, os níveis de vacinação continuem eficazes e o programa de Imunização efetive-se. ${ }^{(13)}$
Em suma, a imunização caracteriza-se por uma ação que visa à prevenção e promoção de saúde de toda a comunidade, e por isso a dissertação dessa temática é de fundamental importância, assim como de todas as etapas envolvidas: desde a fabricação à aplicação da vacina. Por isso a manutenção da qualidade de todo esse sistema é indispensável para que a imunização atinja seu objetivo, ou seja, prevenir e/ou minimizar os agravos à saúde decorrentes das doenças imunopreveníveis.

\section{CONSIDERAÇÕES FINAIS}

Neste estudo, evidenciou-se que as recomendações previstas no Manual de Rede de Frio não estão em sua total conformidade com a prática de conservação de vacinas nas UBSs do município, bem como o serviço local apresenta deficiências que podem interferir na efetividade do Programa Nacional de Imunização.

Essa análise pôde representar um instrumento para a reordenação da sala de vacina e rede de frio do município de Mossoró/RN, reconhecendo que o controle rigoroso das condições de conservação e organização das vacinas, assim como as práticas profissionais são imprescindíveis para assegurar a qualidade e a efetividade da imunização.

Como limitações do estudo cita-se a delonga para fornecer a anuência, o que resultou no atraso para início da pesquisa, a greve dos serviços municipais de saúde no período de coleta de dados e as adversidades vistas com relação às salas de vacina.

Diante de todo o exposto, garantir que a conservação das vacinas seja mantida desde a fabricação dos imunobiológicos até a utilização nos serviços de saúde exige além de equipamentos, o conhecimento técnico dos profissionais envolvidos no processo de conservação e organização dos imunobiológicos. Ressaltando a importância do papel da gestão em providenciar materiais essenciais para consumo, assim como realizar capacitações tais como o treinamento, em sala de vacina e gerenciamento em rede de frio com o intuito de garantir um serviço íntegro a população. 


\section{REFERÊNCIAS}

I. Zinelli AGV, Marcelino DM,Tibola ES, Gôes FC, Silva RF, Melo FAO, Silva F. Imunização na Atenção Básica:Ações do Enfermeiro/Immunization in Primary Care: Nurse's Actions. Id on Line Rev. Mult. Psic, 2019 out. 13 (47). 499-507. Disponível em: https://idonline.emnuvens.com.br/id/article/ view/2104/3192

2. Ministério da Saúde (Brasil), Secretaria de Vigilância em Saúde, Departamento de Vigilância das Doenças Transmissíveis. Manual de Rede de Frio do Programa Nacional de Imunizações / - 5. ed. - Brasília: Ministério da Saúde, 2017. ISBN 978-85-334-2534-7 Disponível em: https:// www.saude.gov.br/images/pdf/20 I 7/dezembro/I5/rede_ frio_2017_web_VF.pdf

3. Brasil. Decisão $N^{\circ} 42$ de 24 de maio de 2018 , dispõe acerca dos procedimentos para diminuição de risco biológico e de infecção cruzada nas salas de vacinação. Diário Oficial da União. Brasília, DF 29 de mai. 2018. Seção I, p. I9I. Disponível em: http://www.in.gov.br/materia/-/asset_publisher/ Kujrw0TZC2Mb/content/id/I6288377/dol-20 I8-05-29decisao-n-42-de-24-de-maio-de-20I8-I6288373

4. Ministério da Saúde (Brasil), Secretaria DeVigilância Em Saúde. Departamento De Vigilância Epidemiológica. Programa Nacional De Imunizações. Programa de avaliação do instrumento de supervisão sala de vacinação - PAISSV. Brasília: Ministério da saúde. 2004. Disponível em: http://pni.datasus. gov.br/Download/Paissv/PAISSV-Instrumento.doc. Acesso em: 02 nov. 2016.

5. Yuzawa LS, Ferreira WFS, Oliveira EM. Políticas Públicas Brasileira de Imunização e Educação Permanente: Um Recorte Temporal Bioético/Brazilian Public Policies on Immunization and Permanent Education: A Temporary Bioethic Cutting Id on Line Rev. Mult. Psic. 2019.13 (45). 95-II0. Disponível em: https://idonline.emnuvens.com.br/id/article/ view/ I 68I/2487 Acesso em 02 de abril de 2020.

6. Ministério da Saúde (Brasil). Secretaria de Vigilância em Saúde. Departamento de Vigilância das Doenças Transmissíveis. Manual de normas e procedimentos para vacinação. Brasília: Ministério da Saúde, 2014. Disponível em: http://bvsms. saude.gov.br/bvs/publicacoes/manual_procedimentos_vacinacao.pdf. Acesso em: I3 ago. 2016.

7. Brasil. (República Federativa do Brasil). Regulamentação do exercício da enfermagem. Lei $n^{\circ} 7.498$, de 25 de junho de 1986. Disponível em: http://www.planalto.gov.br/ccivil_03/ leis/l7498.htm. Acesso em: 10 maio. 2017.

8. Brasil. Resolução COFEN $n^{\circ} 302$ de 16 de março de 2005: aborda a responsabilidade técnica do enfermeiro. Rio de Janeiro. Conselho Federal de Enfermagem, 2005. Disponível em: <http://www.cofen.gov.br/resoluo-cofen-3022005_4337.html>.Acesso em: 17 maio. 2017.

9. Elisiário RN, Siman AG, Moreira TR, Carvalho CA, Amaro MOF. Avaliação das salas de vacinas nas unidades de estratégia de saúde da família. Enferm. Rev; 2017 mar. 20 (3), I-17. Disponível em: http://periodicos.pucminas.br/index. php/enfermagemrevista/article/view/I7228 Acesso em 02 de maio de 2020.

10. Ribeiro AB; Melo CTP; Tavares DRS.A importância da atuação do enfermeiro na sala de vacina: uma revisão integrativa. Rev. de Enferm. da ufjf, 2017 jan/jun. 3 (I) 37-44. Disponível em: https://periodicos.ufjf.br/index.php/enfermagem/ article/view/39/4/1612 Acesso em 03 de maio de 2020.

II. Oliveira VC, Oliveira PP, Castro LC, Rennó HMS, Gonçalves DTA, Pinto IC. Construção coletiva de bundle para boas práticas de conservação de imunobiológicos. Rev; Bras. de Enferm, 2019 mai/jun. 72 (3), 67l-679. Disponível em: https://www.scielo.br/scielo.php?script=sci_arttext\&pi$d=$ S0034-7 I 6720 I 900030067 I \&lng=en\&nrm=iso\&tlng=pt Acesso em 03 de maio de 2020.

12. Rocha JP, Barbosa MWA, Loures SLCS.A imunização contra a influenza como cuidado geriátrico: um estudo epidemiológico que avalia o conhecimento do público senil acerca da adesão imunológica e a cobertura vacinal em Muriaé (MG) sob a ótica do profissional de enfermagem. Rev.Cient. Da Faminas, 2016 fev. 7(I) 28-44. Disponível em: http:// periodicos.faminas.edu.br/index.php/RCFaminas/article/ download/265/24I Acesso em 03 de Maio de 2020.

13. Marques FC, Oliva VL, Sampaio CA. A dor necessária da vacinação e suas nuanças-Percepções de familiares. Revista Enfermagem Atual In Derme, set 2019;89 (27). I-6. Disponível em: https://www.revistaenfermagematual.com/index.php/ revista/article/view/59 Acesso em 04 de agosto de 2020

Recebido: 2020-03-|4

Aceito: 2020-07-I5 